not kept pace with increasing diversity in the $\mathrm{PhD}$ pool.

One aspect of the problem concerns the tendency of departments to go out of their way to recruit minority candidates only to replace previous minority representation. In one study of science and engineering departments in 27 universities, of 1,500 faculty hired during 2000-04, only 157 were African American, Latino or Native American. But nearly three out of five of the new hirings merely replaced minority faculty members who had left the institution.

This is the proverbial 'revolving door' in action. Under-represented minorities are brought in as assistant professors, sometimes through special programmes that aim to improve diversity, only to leave shortly thereafter. Universities that focus on recruitment without placing equal emphasis on retention are not going to achieve the diversity that they seek.

Any successful assault on the persistent problems that universities have faced in nurturing diversity is likely to require the active involvement of under-represented minority faculty themselves. But this group is hard to unify, comprising as it does people who hail from disparate backgrounds and who are shaped by different experiences and social pressures. It can be hard to unite such a diverse group behind a single cause, yet their ranks are so small that full participation is crucial.

Discrimination, where it exists, may be hard to prove in individual cases, but can be identified by collecting appropriate, campus-wide data. Universities need to keep careful track not just of how many under-represented minority researchers they hire, but of how long they stay. The 27-university study found that institutions were rarely tracking data about departures alongside data about hiring.

Tenured faculty members must also learn to recognize the modern face of racial discrimination. This can take many subtle forms: neglecting to recommend a minority colleague to speak at a conference, subconsciously assuming that a minority researcher has a lesser role in a collaboration, or failing to recognize the added time a minority assistant professor may spend advising minority students.

The controversy at the Massachusetts Institute of Technology (MIT) over biological engineer James Sherley's tenure (see page 762) has pushed the institute to embark on a new study that will evaluate racial disparities among its faculty. It is a project that MIT has discussed but left undone for years, and a step that could sensibly be followed by other leading universities. It shouldn't take a hunger strike to prod them into action.

\section{Mentors of tomorrow}

\section{Everyone knows bad peer review when they come across it - but too few are nurturing good referees.}

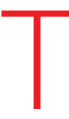
here is nothing more infuriating: you are an experienced scientist who has sent one of your best-ever papers to a journal, and what do you get back? A set of referees' comments that appals you. One reviewer asserts that the work is simply uninteresting and insufficiently original. Another displays wilful bias in relating their criticisms to results by a competitor whose outlook differs radically from yours. And a third has unreasonable expectations of what should be achieved. Not only are you upset, but your student co-author is devastated.

Such a trio of inadequate referees' reports would rightly make an author doubt the credibility of the editor, who has a duty to ensure fair play. But they might also give the offended author reason to reflect a little. What right has he or she to expect a high quality of peer review? What training is being given in his or her own lab to ensure that the next generation understands how to do a good job of critically appraising others' work? And as the pressures on researchers grow - bureaucracy from institutions and funding agencies, incentives to apply the outcomes of research - the very motivation to do a conscientious job of peer review is itself under pressure.

The fact is that the skills required to be a conscientious peer reviewer cannot and should not be taken for granted in young scientists. The culture of peer review is hugely variable - some heads of research groups take such training seriously, others do nothing. A more robust culture of good refereeing needs to be sustained - and that needs strong mentors.

Nature has for some years campaigned for good mentoring in the laboratory, particularly through a set of awards introduced in 2005 (see www.nature.com/npg_/community/nature_awards.html; the entry period for this year's awards, which are for researchers in South Africa, closes this week). On page 791, we publish a guide to all aspects of mentoring, derived from the diverse and very specific endorsements of good practice received through the nominations process.

Many of the goals of mentoring are self-evident. But the explicit fostering of ethics in the lab is rare. And ethical practice includes doing justice to other researchers in critically assessing their work.

So what are the elements of good peer review? The most important aspect is attitude, which should be one of constructive objectivity. This includes not only avoiding scientific bias, but also leaving behind any preconceptions about the labs in which the work was done.

The content should be constructive, too. To summarize the paper and highlight its strengths and essential significance not only shows a proper sense of engagement, but also helps both author and editor to benefit from a fresh perspective. But it is in making critical comment that a referee genuinely adds value, provided that such comments are constructively and collegially expressed. If there are experimental weaknesses or alternative interpretations, it helps to suggest experiments to strengthen the case or resolve ambiguities. If inadequate credit is given to previous work or if the paper fundamentally lacks originality, it is a good idea to provide appropriate references.

This may all seem obvious to the experienced reviewer. (Readers can find more on good peer review, and can comment on it and on this Editorial, at http://blogs.nature.com/peer-to-peer.) But it is only by careful oversight of a young scientist's attempts at reviewing real papers that the benefits of this experience can be passed on. Nature and Nature journals encourage the involvement of younger scientists by a referee, provided the authors' need for confidentiality is respected and the additional reviewer identified. Such mentoring should be routine in research team leadership, if standards are to be kept high. 